\title{
Maternal obesity and gestational weight gain: associations with maternal and infant outcomes
}

\author{
L. Cassidy¹, M.S. Mulhern¹, R.M. Alhomaid¹, M.B.E. Livingstone1, J.C. Abayomi² and M.T. McCann¹ \\ ${ }^{1}$ Nutrition Innovation Centre for food and HEalth (NICHE), Ulster University, Coleraine, BT52 1SA, ${ }^{2}$ Faculty of Education, Health and \\ Community, Liverpool John Moores University, L17 6BD.
}

\section{Introduction}

- Obesity among women of childbearing age has become a major public health issue with one in five women now entering pregnancy classified as obese

- Additionally, excessive gestational weight gain (GWG) has been implicated as a risk factor for pregnancy complications and can lead to retained weight postpartum and increased rates of obesity ${ }^{2}$

- Currently, there are no evidence-based UK guidelines for weight-gain during pregnancy, with the routine weighing of women being discouraged ${ }^{3}$

Aim

- To examine the influence of BMI and GWG on maternal and infant birth outcomes across normal weight, overweight and obese pregnant women

\section{Methods}

- Data were collected from an ongoing observational study in normal weight, overweight and obese pregnant women

- A total of 240 pregnant women without current complications, aged $\geq 18$ years and having a singleton pregnancy were recruited

- Maternal weight $(\mathrm{kg})$ was measured at three time points

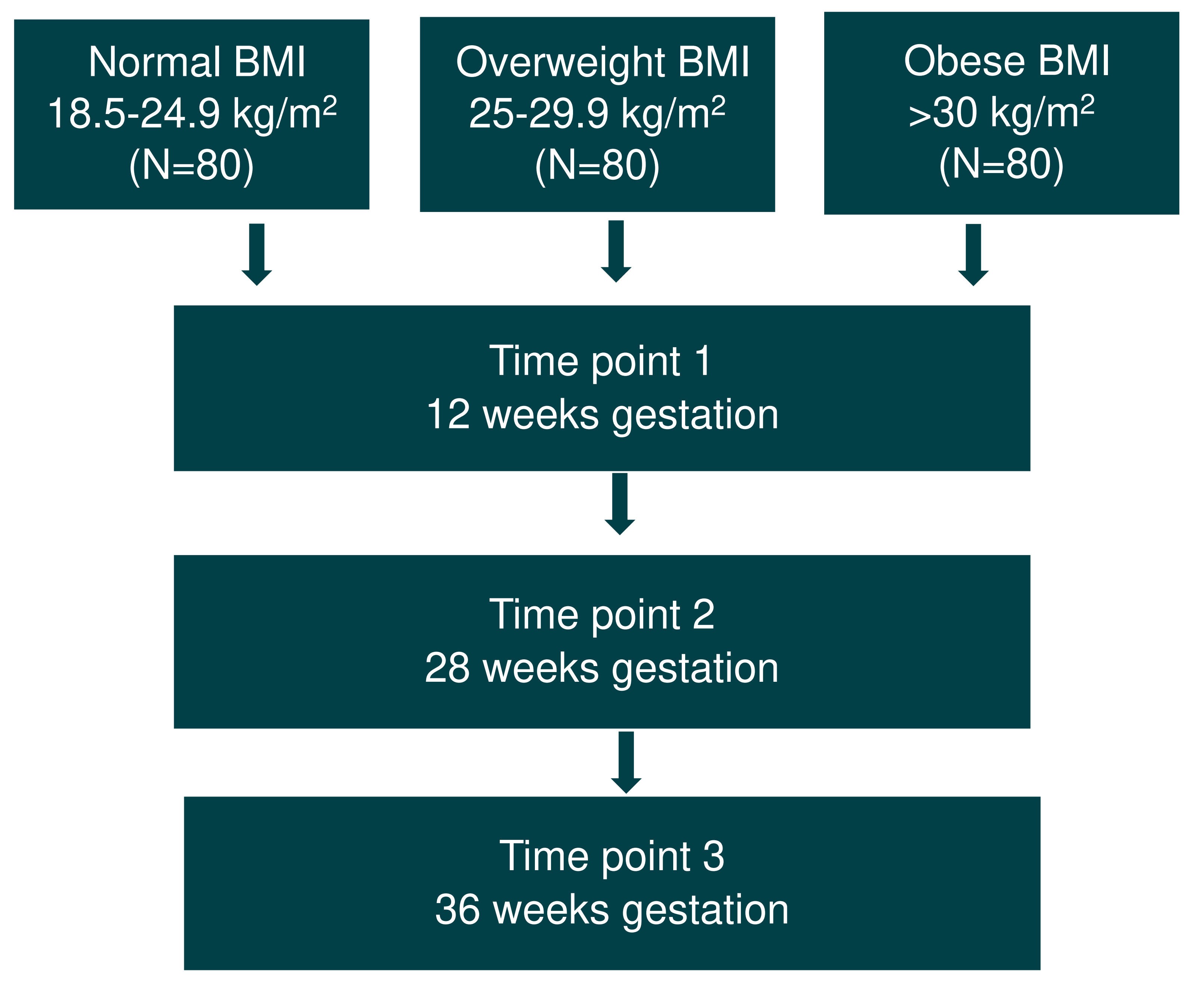

- Infant anthropometric measures at birth and other maternal and infant outcomes were recorded from maternal/paediatric notes

- GWG and birth outcomes were assessed, across and within BMI groups

- GWG was calculated for each BMI group and classified as either low or high GWG according to being below or above the median within that BMI group

- Data were not-normally distributed; the non-parametric Mann-Whitney $U$, Chi square and Kruskal Wallis tests were used as appropriate

\section{Results}

Table 1. Maternal baseline characteristics in normal weight, overweight and obese pregnant women

\begin{tabular}{|l|c|c|c|c|} 
& $\begin{array}{c}\text { Normal } \\
\mathbf{N = 8 0}\end{array}$ & $\begin{array}{c}\text { Overweight } \\
\mathbf{N = 7 9}\end{array}$ & $\begin{array}{c}\text { Obese } \\
\mathbf{N = 8 0}\end{array}$ & P value \\
\hline Age $(\mathrm{yrs})$ & $31.0(25.2,33.7)$ & $30.0(26.0,34.0)$ & $30.0(25.0,32.0)$ & 0.529 \\
\hline Weight $(\mathrm{kg})$ & $60.0(54.5,64.2)^{\mathrm{a}}$ & $71.7(67.1,77.4)^{\mathrm{b}}$ & $88.6(83.2,99.6)^{\mathrm{c}}$ & $<\mathbf{0 . 0 0 0 1}$ \\
\hline Height $(\mathrm{m})$ & $1.63(1.58,1.68)$ & $1.63(1.58,1.68)$ & $1.63(1.59,1.67)$ & 0.947 \\
\hline $\begin{array}{l}\text { Weeks gestation } \\
\text { Smoker } \mathrm{n}(\%)\end{array}$ & $13.0(12.0,13.6)$ & $13.0(12.0,13.6)$ & $12.6(12.1,13.4)$ & 0.945 \\
\hline $\begin{array}{c}\text { Parity } \mathrm{n}(\%) \\
0\end{array}$ & $8(10)$ & $11(13.8)$ & $11(14.1)$ & 0.679 \\
\hline 1 & $28(35.4)$ & $35(44.3)$ & $33(42.3)$ & 0.826 \\
\hline $2+$ & $30(38)$ & $25(31.3)$ & $27(34.6)$ & \\
\hline
\end{tabular}

Data presented as median (IQR) or $n$ (percentage). Kruskal Wallis and Chi square test used to compare
differences between BMI groups. Values in a row with different superscript letters indicate significant

Table 2. Total GWG in normal weight, overweight and obese pregnant women

\begin{tabular}{|l|c|c|c|c|}
\hline & $\begin{array}{c}\text { Normal } \\
\mathbf{N = 5 7}\end{array}$ & $\begin{array}{c}\text { Overweight } \\
\mathbf{N = 4 8}\end{array}$ & $\begin{array}{c}\text { Obese } \\
\mathbf{N = 5 1}\end{array}$ & P value \\
\hline Total GWG $(\mathrm{kg})$ & $11.4(9.0,13.4)^{\mathrm{a}}$ & $8.1(6.6,12.2)^{\mathrm{b}}$ & $8.8(3.6,11.7)^{\mathrm{b}}$ & $\mathbf{0 . 0 0 4}^{*}$
\end{tabular}
Data presented as median (IQR), Kruskal Wallis test used to compare differences between BMI groups.
Values in a row with different superscript letters indicate significant difference $(P<0.05) P<0$.

$70 \%$

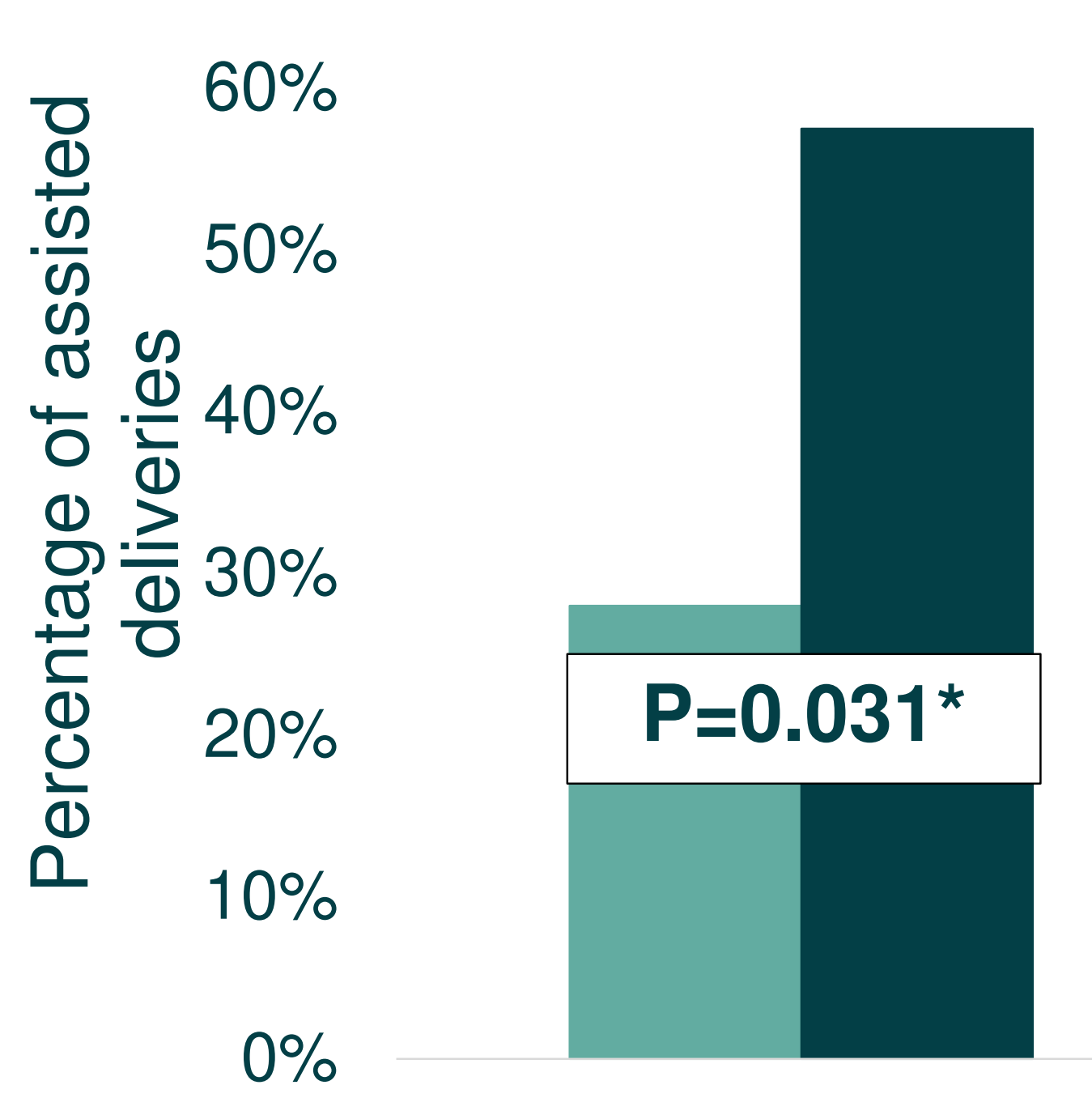

Normal weight

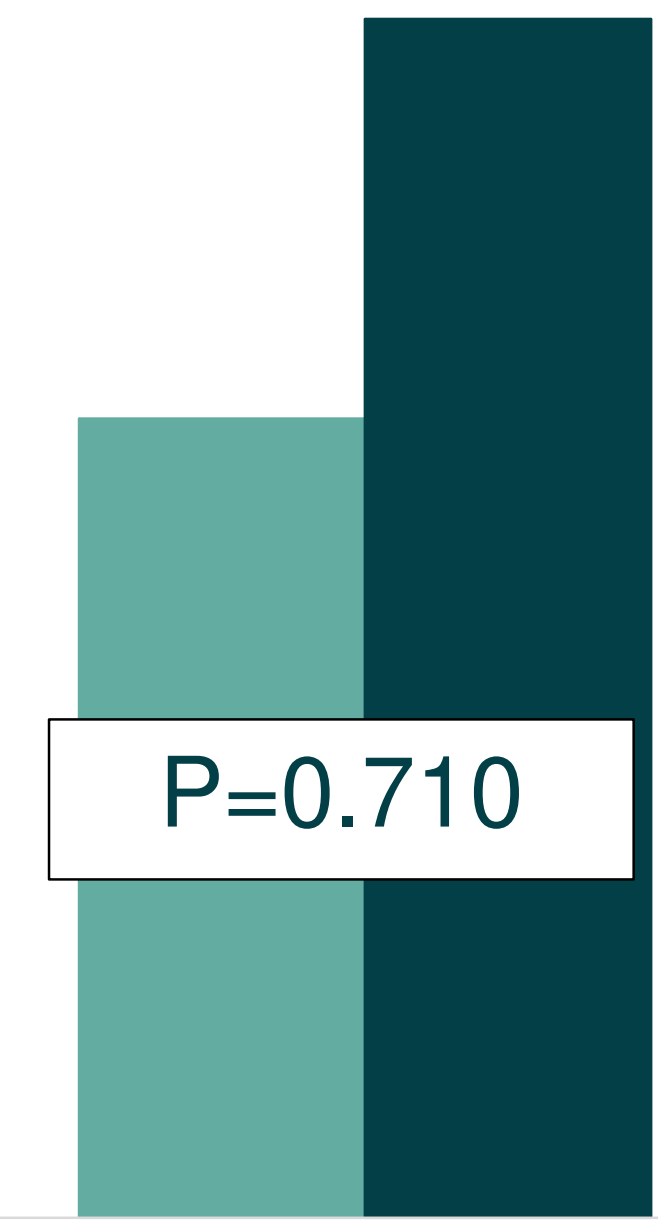

Overweight
Low GWG

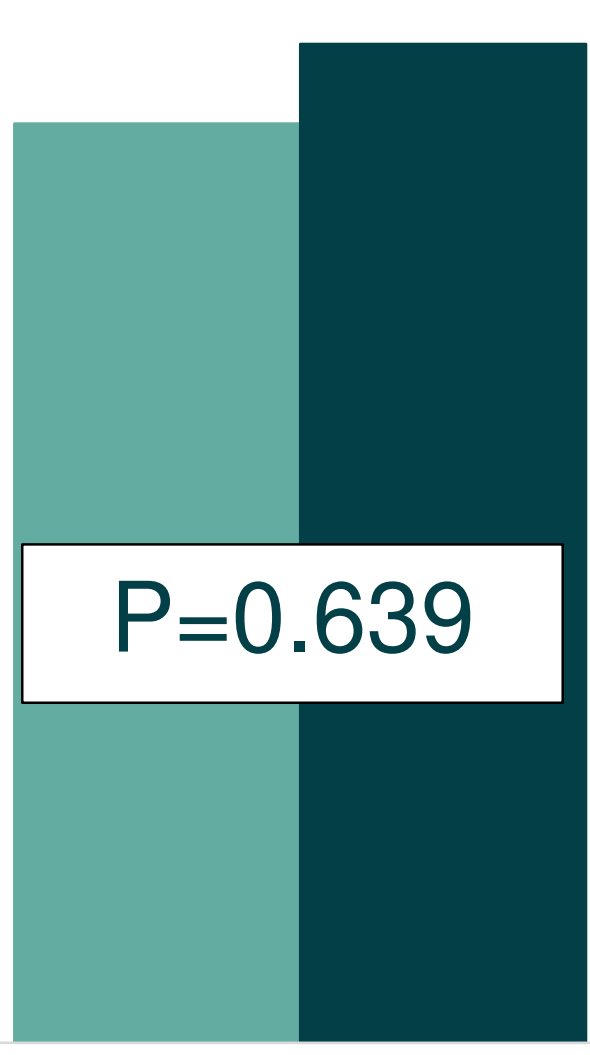

Obese
Figure 1: Delivery outcomes comparing high to low GWG within normal weight, overweight and obese categories

- Normal weight women who were in the high GWG group required significantly more assisted deliveries than normal weight women in the low GWG group

- These differences were not observed in the overweight or obese group

\section{Conclusions}

- These findings demonstrate that in normal weight women, a higher GWG is associated with an increased need for assisted delivery

- This research highlights the need for revised maternal policy on the monitoring of weight gain during pregnancy with the view to improving maternal and infant outcomes

\section{References}

1. NHS digital (2017) - Statistics on obesity, physical activity and diet https://www.gov.uk/government/uploads/system/uploads/attachment data/file/613532/ obes-phys-acti-diet-eng-2017-rep.pdf

2. Lipsky LM, Strawderman MS et al. Obesity;2015 20(7)1496-150

3. NICE (2010)- Weight management before, during and after pregnancy. https://www.nice.org.uk/guidance/ph27/resources/guidance-weight-managementbefore-during-and-after-pregnancy-pdf 